\title{
A Numerical Solution Method for an Infinitesimal Elasto-Plastic Cosserat Model
}

\author{
Wolfgang Müller ${ }^{1}$, Patrizio Neff ${ }^{2}$, Christian Wieners ${ }^{1}$ \\ ${ }^{1}$ Fachbereich Mathematik, Universität Karlsruhe (TH) \\ ${ }^{2}$ Fachbereich Mathematik, Technische Universität Darmstadt
}

GAMM 2008 in Bremen

Session 8: Multiscales and homogenization

April 1, 2008 


\section{A micropolar extension to infinitesimal elasticity}

- We present a geometrically linear generalized continua of Cosserat micropolar type in the elasto-plastic case.

- We postulate independent infinitesimal microrotations of the material. Thus, as a consequence of balance of angular momentum, Cauchy stresses $\sigma$ are not symmetric any more.

- Cosserat effects regularize the mesh size dependence of localization computations where shear failure mechanisms play a dominant role.

- We restrict Cosserat microrotations to the elastic response of the material. Inelasticity is formulated as in Prandtl-Reuß plasticity. The elasto-plastic Cosserat problem is well-posed (Neff/Chełmiński Appl.Math.Opti06, PRSE05). 


\section{Infinitesimal time continuous Cosserat Plasticity}

Let $\Omega \subset \mathbb{R}^{3}$ be the reference configuration, and let $\Gamma_{D} \cup \Gamma_{N}=\partial \Omega$ be a decomposition of the boundary.

We consider infinitesimal microrotations $\bar{A} \in \mathfrak{s o}(3):=\left\{\bar{B} \in \mathbf{R}^{3,3}: \bar{B}^{T}=-\bar{B}\right\}$, and we define the symmetric bilinear form

$$
\begin{aligned}
& a\left(\left(\mathbf{u}, \bar{A}, \varepsilon_{p}\right),(\mathbf{v}, \bar{B}, \eta)\right)= \\
& 2 \mu \int_{\Omega}\left(\operatorname{sym}\left(D \mathbf{u}-\varepsilon_{p}\right): \operatorname{sym}(D \mathbf{v}-\eta)\right) d \mathbf{x}+\lambda \int_{\Omega} \operatorname{tr} D \mathbf{u} \cdot \operatorname{tr} D \mathbf{v} d \mathbf{x} \\
& +2 \mu_{c} \int_{\Omega}(\operatorname{skew}(D \mathbf{u}-\bar{A}): \operatorname{skew}(D \mathbf{v}-\bar{B})) d \mathbf{x}+2 \mu L_{c}^{2} \int_{\Omega} D \bar{A}: D \bar{B} d \mathbf{x},
\end{aligned}
$$

where $\mu_{c}$ is the Cosserat couple modulus and $L_{c}$ the internal length scale.

Here, no physical interpretation of Cosserat parameters!

The goal is regularization through elastic size effect. 
Furthermore, we have the convex functional

$$
j\left(\varepsilon_{p}\right)=\int_{\Omega} K_{0}\left|\varepsilon_{p}\right| d \mathbf{x},
$$

(with $\varepsilon_{p} \in\left\{\eta \in \mathbf{R}^{d, d}: \eta^{T}=\eta, \operatorname{tr} \eta=0\right\}$ ), and the load functional

$$
\ell(t, \mathbf{v})=\int_{\Omega} \mathbf{b}(t) \cdot \mathbf{v} d \mathbf{x}+\int_{\Gamma_{N}} \mathbf{t}_{N}(t) \cdot \mathbf{v} d \mathbf{a} .
$$

Quasi-static infinitesimal Cosserat plasticity is characterized by the variational inequality

$$
\begin{array}{r}
a\left(\left(\mathbf{u}, \bar{A}, \varepsilon_{p}\right),(\mathbf{v}, \bar{B}, \eta)-\left(\dot{\mathbf{u}}, \dot{\bar{A}}, \dot{\varepsilon}_{p}\right)\right)+j(\eta)-j\left(\dot{\varepsilon}_{p}\right) \geq \ell(\mathbf{v}-\dot{\mathbf{u}}), \\
\text { for all }(\mathbf{v}, \bar{B}, \eta) \in \mathbf{V} \times W \times \mathbf{E},
\end{array}
$$

subject to boundary conditions and given material history $\varepsilon_{p}(0)=\varepsilon_{p}^{0}$ as initial value, where $\mathbf{V} \times W \times \mathbf{E}$ is a suitable function space. 


\section{Infinitesimal Elasto-Plastic Cosserat Model - Equations}

We want to determine displacements

$\mathbf{u}: \quad \bar{\Omega} \times[0, T] \longrightarrow \mathbb{R}^{3}$,

infinitesimal micro-rotations non-symmetric stresses symmetric plastic strains and a plastic multiplier

$\bar{A}: \quad \Omega \times[0, T] \longrightarrow \mathfrak{s o}(3)$,

$\boldsymbol{\sigma}: \quad \Omega \times[0, T] \longrightarrow \mathbf{R}^{3,3}$,

$\varepsilon_{p}: \quad \Omega \times[0, T] \longrightarrow \operatorname{Sym}(3)$ with $\varepsilon_{p}(0)=\mathbf{0}$,

$\Lambda: \quad \Omega \times[0, T] \longrightarrow \mathbb{R}$,

satisfying the essential boundary conditions and the equilibrium equations

$$
\begin{array}{llll}
-\operatorname{div} \boldsymbol{\sigma}(\mathbf{x}, t) & =\mathbf{b}(\mathbf{x}, t), & & (\mathbf{x}, t) \in \Omega \times[0, T], \\
\boldsymbol{\sigma}(\mathbf{x}, t) \mathbf{n}(\mathbf{x}) & =\mathbf{t}_{N}(\mathbf{x}, t), & & (\mathbf{x}, t) \in \Gamma_{N} \times[0, T], \\
-\mu L_{c}^{2} \Delta \bar{A}(\mathbf{x}, t) & =\mu_{c}(\operatorname{skew}(D \mathbf{u}(\mathbf{x}, t))-\bar{A}(\mathbf{x}, t)), & & (\mathbf{x}, t) \in \Omega \times[0, T], \\
D \bar{A}(\mathbf{x}, t) \cdot \mathbf{n}(\mathbf{x}) & =\mathbf{0}, & & (\mathbf{x}, t) \in \Gamma_{N} \times[0, T],
\end{array}
$$

the constitutive relation

$$
\begin{aligned}
\boldsymbol{\sigma}(\mathbf{x}, t)= & 2 \mu\left(\operatorname{sym}(D \mathbf{u}(\mathbf{x}, t))-\varepsilon_{p}(\mathbf{x}, t)\right)+\lambda \operatorname{tr} D \mathbf{u}(\mathbf{x}, t) \cdot \mathbf{I} \\
& +2 \mu_{c}(\operatorname{skew}(D \mathbf{u}(\mathbf{x}, t))-\bar{A}(\mathbf{x}, t)), \quad(\mathbf{x}, t) \in \Omega \times[0, T],
\end{aligned}
$$




\section{Infinitesimal Elasto-Plastic Cosserat Model - Equations}

the complementary conditions for the yield criterion

$\Lambda(\mathbf{x}, t) \phi\left(T_{E}(\mathbf{x}, t)\right)=0, \quad \Lambda(\mathbf{x}, t) \geq 0, \quad \phi\left(T_{E}(\mathbf{x}, t)\right) \leq 0, \quad(\mathbf{x}, t) \in \Omega \times[0, T]$.

and the flow rule

$$
\frac{d}{d t} \varepsilon_{p}(\mathbf{x}, t)=\Lambda(\mathbf{x}, t) D \phi\left(T_{E}(\mathbf{x}, t)\right), \quad(\mathbf{x}, t) \in \Omega \times[0, T],
$$

depending on $T_{E}(\mathbf{x}, t)=2 \mu\left(\operatorname{sym}(D \mathbf{u}(\mathbf{x}, t))-\varepsilon_{p}(\mathbf{x}, t)\right)$.

For given material history $\varepsilon_{p}(t)$ at fixed time $t$, the displacement and the micro-rotations are determined by minimizing the total elastic energy

$$
\mathcal{I}\left(\mathbf{u}, \bar{A}, \varepsilon_{p}\right)=\mathcal{E}\left(\varepsilon(\mathbf{u}), \bar{A}, \varepsilon_{p}\right)-\ell(t, \mathbf{u}),
$$

with $\mathcal{E}\left(\varepsilon, \bar{A}, \varepsilon_{p}\right)=\mu \int_{\Omega}\left|\operatorname{sym}(\varepsilon)-\varepsilon_{p}\right|^{2} d \mathbf{x}+\frac{\lambda}{2} \int_{\Omega} \operatorname{tr}(\varepsilon)^{2} d \mathbf{x}$

$+\mu_{c} \int_{\Omega}|\operatorname{skew}(\varepsilon)-\bar{A}|^{2} d \mathbf{x}+\mu L_{c}^{2} \int_{\Omega}|D \bar{A}|^{2} d \mathbf{x}$. 


\section{Discrete formulation of the Elasto-Plastic Model}

Let $\mathbf{V}_{h} \times W_{h}$ be a finite element space with $\mathbf{v}_{h}=\mathbf{0}$ and $\bar{B}_{h}=0$ on $\Gamma_{D}$. The model of incremental infinitesimal plasticity is obtained by a decomposition $0=t_{0}<t_{1}<\cdots<t_{N}=T$ of the time interval and backward Euler scheme.

\section{Lemma:}

The fully discrete elasto-plastic problem is equivalent to the following nonlinear weak problem. For given $\varepsilon_{p}^{n-1}$ find $\left(\mathbf{u}^{n}, \bar{A}^{n}\right) \in \mathbf{V}_{h} \times W_{h}$ such that

$$
\begin{aligned}
& \int_{\Omega} P_{\mathbf{K}}\left(2 \mu\left(\operatorname{sym}\left(D \mathbf{u}_{h}^{n}\right)-\varepsilon_{p}^{n-1}\right)\right): D \mathbf{v}_{h} d \mathbf{x}+\lambda \int_{\Omega} \operatorname{tr} D \mathbf{u}_{h}^{n} \cdot \operatorname{tr} D \mathbf{v}_{h} d \mathbf{x} \\
& \quad+2 \mu_{c} \int_{\Omega}\left(\operatorname{skew}\left(D \mathbf{u}_{h}^{n}\right)-\bar{A}_{h}^{n}\right): D \mathbf{v}_{h} d \mathbf{x}=\ell\left(t_{h}, \mathbf{v}_{h}\right), \quad \mathbf{v}_{h} \in \mathbf{v}_{h}, \\
& \mu L_{c}^{2} \int_{\Omega} D \bar{A}_{h}^{n} \cdot D \bar{B}_{h} d \mathbf{x}=\mu_{c} \int_{\Omega}\left(\operatorname{skew}\left(D \mathbf{u}_{h}^{n}\right)-\bar{A}_{h}^{n}\right): \bar{B}_{h} d \mathbf{x}, \quad \bar{B}_{h} \in W_{h},
\end{aligned}
$$

with the orthogonal projection $P_{\mathbf{K}}(\boldsymbol{\theta})=\boldsymbol{\theta}-\max \left\{0,|\operatorname{dev}(\boldsymbol{\theta})|-K_{0}\right\} \frac{\operatorname{dev}(\boldsymbol{\theta})}{|\operatorname{dev}(\boldsymbol{\theta})|}$ on the elastic domain $\mathbf{K}:=\left\{\boldsymbol{\tau} \in \mathbf{R}^{3,3}: \boldsymbol{\tau}^{T}=\boldsymbol{\tau},|\operatorname{dev} \boldsymbol{\tau}| \leq K_{0}\right\}$ of the von Mises flow rule. 


\section{Variational Formulation of the discrete problem}

\section{Lemma:}

Any minimizer $\left(\mathbf{u}_{h}^{n}, \bar{A}_{h}^{n}\right) \in \mathbf{V}_{h} \times W_{h}$ of the functional

$$
\mathcal{I}_{\text {incr }}^{n}\left(\mathbf{u}_{h}, \bar{A}_{h}\right)=\mathcal{E}_{\text {incr }}\left(D \mathbf{u}_{h}, \bar{A}_{h}, \varepsilon_{p}^{n-1}\right)-\ell\left(t_{n}, \mathbf{u}_{h}\right)
$$

solves the nonlinear variational update problem. Here $\mathcal{E}_{\text {incr }}$ denotes the free energy of the incremental update problem defined by

$$
\begin{aligned}
& \mathcal{E}_{\text {incr }}\left(D \mathbf{u}, \bar{A}, \varepsilon_{p}\right)=\frac{1}{2 \mu} \int_{\Omega} \psi_{\mathbf{K}}\left(2 \mu\left(\operatorname{sym}(D \mathbf{u})-\varepsilon_{p}\right)\right) d \mathbf{x}+\frac{\lambda}{2} \int_{\Omega} \operatorname{tr}(D \mathbf{u})^{2} d \mathbf{x} \\
& +\mu_{c} \int_{\Omega}|\operatorname{skew}(D \mathbf{u})-\bar{A}|^{2} d \mathbf{x}+\mu L_{c}^{2} \int_{\Omega}|D \bar{A}|^{2} d \mathbf{x}, \\
& \psi_{\mathbf{K}}(\boldsymbol{\theta})=\left\{\begin{array}{cl}
\frac{1}{2}|\boldsymbol{\theta}|^{2} & |\operatorname{dev}(\boldsymbol{\theta})| \leq K_{0}, \\
\frac{1}{2}\left(\frac{1}{d} \operatorname{tr}(\boldsymbol{\theta})^{2}+2 K_{0}|\operatorname{dev}(\boldsymbol{\theta})|-K_{0}^{2}\right) & |\operatorname{dev}(\boldsymbol{\theta})|>K_{0} .
\end{array}\right.
\end{aligned}
$$

If $\varepsilon_{p}^{n-1}=0$ and $\mu_{c}=0 \rightarrow$ classical Hencky-Problem. 


\section{The FEM convergence}

\section{Theorem:}

We have

$$
\left\|\left(\mathbf{u}-\mathbf{u}_{h}, \bar{A}-\bar{A}_{h}\right)\right\| \mathbf{v} \times w \leq \frac{C}{\mu_{c}} \inf _{\left(\mathbf{v}_{h}, \bar{B}_{h}\right) \in \mathbf{v}_{h} \times W_{h}}\left\|\left(\mathbf{u}-\mathbf{v}_{h}, \bar{A}-\bar{B}_{h}\right)\right\| \mathbf{v} \times w .
$$

$C$ is independent of $\mu_{C} \in(0, \mu]$.

If the analytical solution $u$ is $H^{2}$-smooth then

$$
\inf _{\left(\mathbf{v}_{h}, \bar{B}_{h}\right) \in \mathbf{V}_{h} \times W_{h}}\left\|\left(\mathbf{u}-\mathbf{v}_{h}, \bar{A}-\bar{B}_{h}\right)\right\| \mathbf{v}_{\times} \times W \leq C h\left(\|u\|_{\mathbf{H}^{2}(\Omega)}+\|\bar{A}\|_{\mathbf{H}^{2}(\Omega)}\right) .
$$

Neff/Knees: Cosserat update problem admits unique global $H^{2}$-solutions! Idea: balance $h$ against $\mu_{c}$. 


\section{Plate with a hole}

Let $\Omega=(0,10) \times(0,10) \backslash B_{1}(10,0)$. We use Q1 discretization and present results for 198147 unknowns on uniform refinement level 4 . We have chosen the parameters $K_{0}=450, \lambda=110743.8, \mu=80193.8$ and $L_{c}=0.020833$. And apply traction force by Neumann boundary condition according to: $\ell(t, v)=100 t \int_{0}^{10} \mathbf{v}\left(x_{1}, 10\right) d x_{1}$.
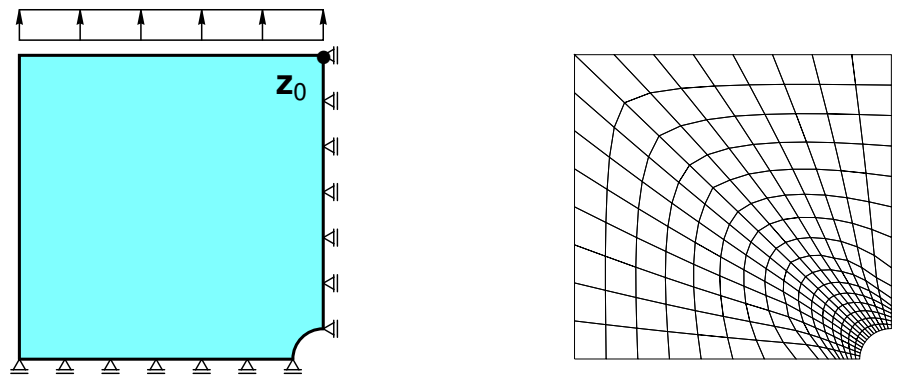

Geometry, boundary conditions and coarse mesh. 


\section{Numerical Experiment with $\mathrm{M}_{++}$}

Cosserat Model $\left(\mu_{c}=\mu\right)$ : Effective plastic strain
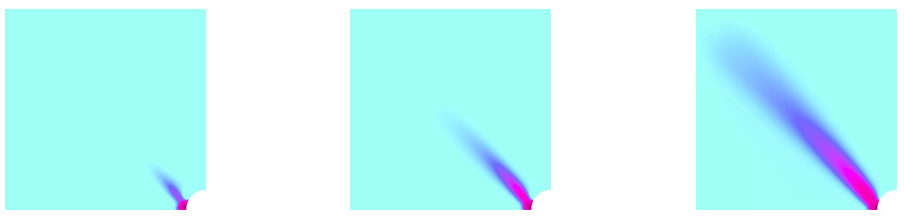

Prandtl-Reuß $\left(\mu_{c}=0\right)$ : Effective plastic strain
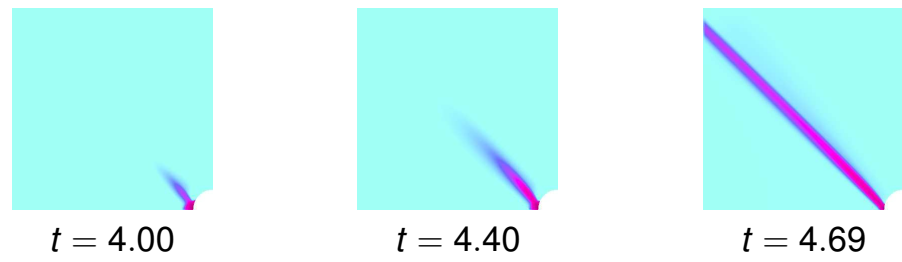
Notched cube - a test configuration in 3D
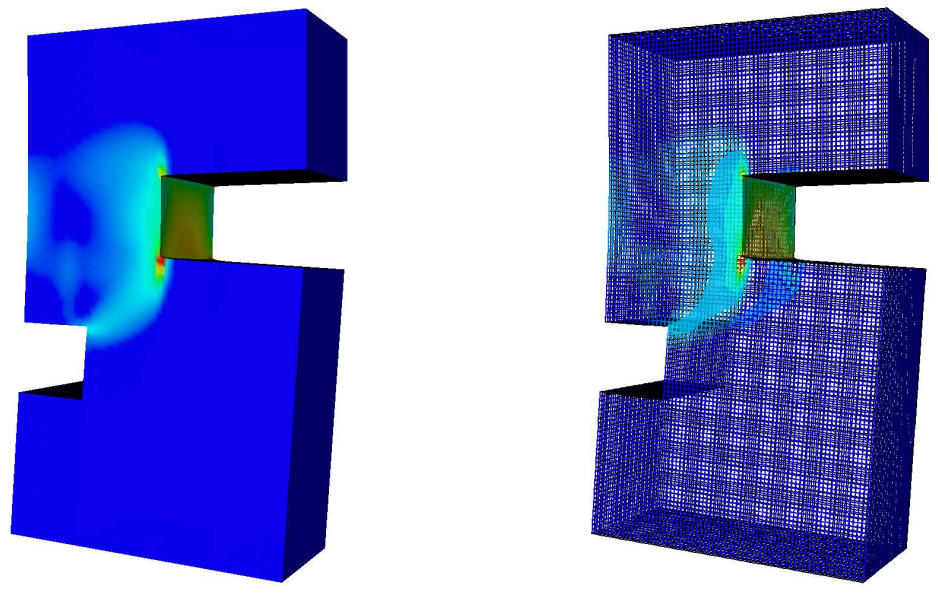

For the linear subproblem in each Newton step, we use a parallel multilevel GMRES algorithm. 


\section{Numerical Experiment with $\mathrm{M}++$}

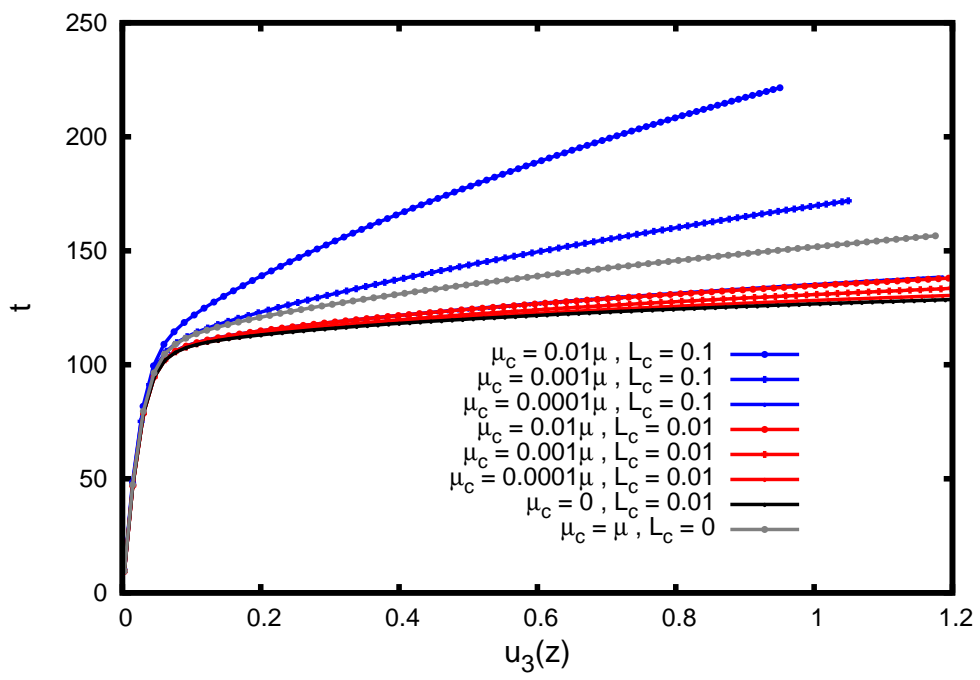

Load-displacement curve for notched cube on refinement level 3. Here, we use 174.727 dof for displacement and microrotation. 


\section{Numerical Experiment with $\mathrm{M}++$}

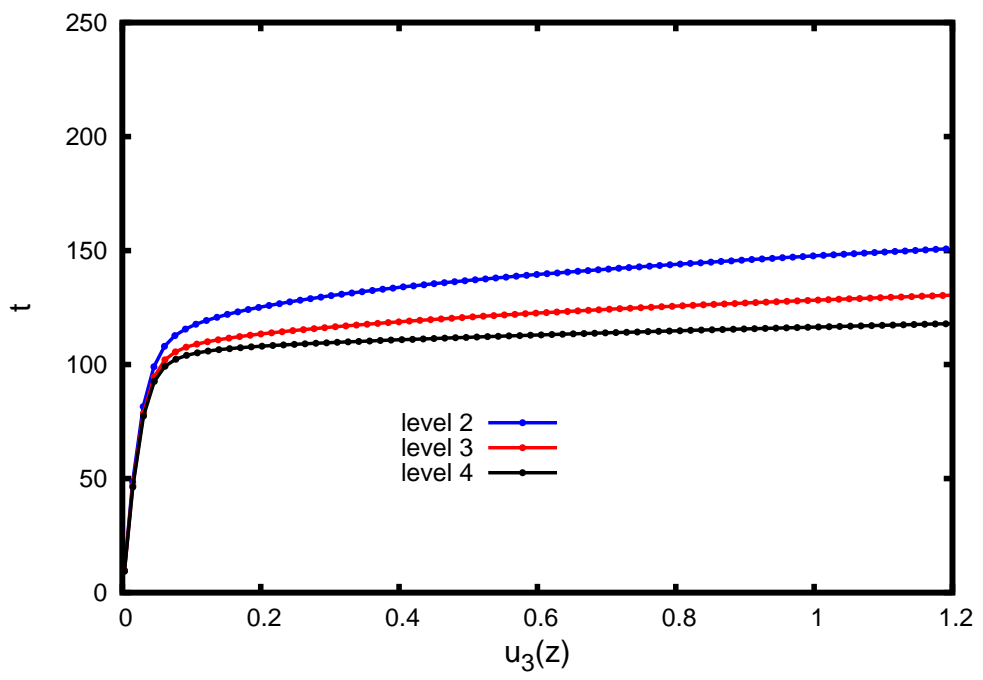

Here, we compute up to 1.311.751 dof on refinement level 2, 3 and 4 $\left(\mu_{c}=0.0001 \mu, L_{c}=0.01\right)$. We observe about linear convergence in $h$. 


\section{Summary and Outlook}

- The Elasto-Plastic Cosserat Model with pure Dirichlet data is well-posed: unique solution globally in time.

- The discrete update problem admits global $H^{2}$-solutions.

- For linear Lagrange elements, we have linear convergence in $h$.

- The Elasto-Plastic Cosserat Model is a regularization for classical perfect plasticity (shear failure mechanisms).

- Future work will be the analysis and robust implementation of geometrically nonlinear elasto-plastic Cosserat Models. 


\section{Selected References}

- P. Neff, K. Chełmiński, W. Müller and C. Wieners, A numerical solution method for an infinitesimal elastic-plastic Cosserat model, Math. Mod. Meth. Appl. Sci. (M3AS), 17, 1211-1239, 2007, IWRMM - preprint Nr. 06/10 http://www.mathematik.uni-karlsruhe.de/iwrmm

- P. Neff and D. Knees, Regularity up to the boundary for nonlinear elliptic systems arising in time-incremental infinitesimal elasto-plasticity, to appear in SIAM J. Math. Anal.

- P. Neff, A. Sydow and C. Wieners, Numerical approximation of incremental infinitesimal gradient plasticity, submitted to Int. J. Num. Meth. Engng. IWRMM - preprint Nr. 08/01 http://www.mathematik.uni-karlsruhe.de/iwrmm

- P. Neff and K. Chełmiński, Infinitesimal elastic-plastic Cosserat micropolar theory. Modelling and global existence in the rate independent case, Proc. Roy. Soc. Edinb. A, 135, 1017-1039, 2005

- P. Neff and K. Chełmiński, Well-posedness of dynamic Cosserat plasticity, Appl. Math. Optim., 56, 19-35, 2007 